THE NATURE OF THE ENERGY SOURCE IN RADIO GALAXIES AND ACTIVE GALACTIC NUCLEI

F. Pacini, Arcetri Astrophysical observatory and University of Florence, Italy

M. Salvati, Institute of Space Astrophysics CNR CNR Frascati, Italy

THE ENERGY REQUIREMENTS

For more than 20 years it has been known that extragalactic radio sources contain up to $10^{60}-10^{62}$ ergs in the form of relativistic electrons and magnetic fields. One arrives at these figures if one assumes that the radio emission is due to the synchrotron process and the source contains an equal amount of energy in electrons and fields (Burbidge 1956). Any deviation from the postulated equipartition increases the energy required to account for the observed luminosities. Some authors believe that the real demands on the energy source may be still higher because of the probable presence of high energy protons. The ratio $\mathrm{E}_{\mathrm{p}} / \mathrm{E}_{\mathrm{e}}$ is determined by the way in which particles gain and lose energy, and it is impossible to estimate it a priori. Observationally one has two conflicting lines of evidence: (a) in galactic cosmic rays one measures $\left(\mathrm{E}_{\mathrm{p}} / \mathrm{E}_{\mathrm{e}}\right) \simeq 10^{2} ;(\mathrm{b})$ in the Crab Nebula one infers $\left(E_{p} / E_{e}\right) \lesssim 1$ (otherwise the dynamical pressure of the proton gas would cause a nebular expansion much faster than observed).

A totally different way to estimate the energy requirements in active galactic nuclei consists in multiplying the luminosity across the electromagnetic spectrum (up to, say, $10^{47}$ erg $\mathrm{s}^{-1}$ ) by the average lifetime deduced from statistical arguments ( $10^{8}$ years?). Again one obtains yields reaching $10^{62} \mathrm{erg}$. We note that this figure is the equivalent of about $10^{8} \mathrm{M}_{\Theta} \mathrm{c}^{2}$.

\title{
THE SOURCE OF ENERGY
}

Many processes, both conventional and exotic, have been invoked in order to account for the source of energy. Only the conventional models have been worked in some detail while the exotic ones (quarks' fusion, white holes, etc.) have remained in the stage of proposals. At present, most people agree that gravity is the most likely cause of the energy supply. Indeed, its efficiency can be larger than that of 
nuclear fusion (roughly $40 \%$ against $1 \%$ ), and objects are actually seen where gravitational energy is released in the form of high-energy particles and photons (e.g., supernovae, pulsars, strong binary X-ray sources).

There are two different classes of models based upon the conversion of gravitational energy.

(a) Very dense clusters of $1-10 \mathrm{M}_{0}$ stars. The stars are assumed to have frequent collisions deep in the galactic nucleus, or to ignite a chain of SN explosions, or perhaps to be already in the form of pulsars. The rationale of this approach is to gather in a small volume of space a collection of stellar-size gravitational engines, each capable of $\lesssim 1053 \mathrm{erg}$, and to add their individual outputs.

(b) One (or, at most, a few) supermassive objects. Their mass would have to be of the order of $10^{8}-10^{9} \mathrm{M}_{\odot}$ and their radius close to the gravitational radius $R_{S} \simeq 10^{13}\left(\mathrm{M} / 10^{8} \mathrm{M}_{\odot}\right) \mathrm{cm}$ in order to meet the energy requirements.

In our opinion the observational evidence collected in the last several years leaves little room for models of class (a). Superimposed on the average emission, there are indeed sudden outbursts of luminosity at various wavelengths. These outbursts of ten involve more than $10^{53}$ erg, occasionally much more (Angel and Stockman 1980; Jones and Burbidge 1973; Stein et al. 1976). Their energetics cannot be accommodated in the framework of stellar collisions or individual SN explosions, and some kind of collective behavior should be postulated, which would change class a models into class b. On the other hand, in the case of supermassive objects with a binding energy $\simeq 10^{62} \mathrm{erg}$, it is hardly difficult for the ingenuity of theorists to invent mechanisms which can release suddenly the required amount of energy (e.g., instabilities in the object's magnetosphere).

The nature of the central supermassive body is unknown, but at present many researchers favor the idea that it might be a black hole, following the original suggestion by Salpeter (1964; for more recent contributions, see Rees 1980). Others have argued that the central body could be a magnetoid (Ginzburg and Ozernoy 1977) or perhaps a rotationally supported spinar (Cavaliere et al. 1969; Morrison 1969; Woltjer 1971). From many points of view the observational consequences of these configurations are the same and there are no critical

arguments in favor of any of them. It is important to stress that all dynamical evidence for a central density enhancement in some galactic nuclei (e.g., M87) cannot distinguish black holes from other configurations, provided these are compact but not necessarily collapsed anywhere near the gravitational radius. However, if the inevitable fate of all mass condensations above a certain threshold is the collapse into a black hole, then black holes should be more common in galactic nuclei than their relatively short-lived precursors. 
In our opinion the nature of the central object is unclear at present and may remain unclear for some time. As we shall see in the following, it is still profitable to discuss the processes which extract energy from that object without worrying too much about its precise physical state.

\section{ACCRETION VS ELECTRODYNAMIC MODELS}

Once again, two kinds of mechanisms have been proposed for the basic engine:

(a) Accretion, i.e., the direct release of gravitational energy by matter (gas and/or disrupted stars) falling into the central body. The latter only acts as the source of the gravitational field and would remain invisible if no fuel were available.

(b) Electrodynamic models, i.e., the release of energy from a region of size R permeated with strong electromagnetic fields. This can take either the form of a genuine Poynting flux $B^{2} R^{2} c$ in pulsar-type situations, or represent the rate $d / d t\left(B^{2} R^{3}\right)$ at which e.m. fields are burned-out in flare-like phenomena. In either case gravity has done its work at an earlier time by converting potential energy into fast rotation and/or strong fields.

It is well known that both (a) and (b) can work in real 1 ife: Accretion is the basic element for the activity of binary $X$-ray sources, while electrodynamic models are involved in the relationship between rotating neutron stars and SN remnants. In some cases the distinction between the two mechanisms could become rather artificial: For instance, on-going accretion could increase the strength of the magnetic field and deform its geometry, thus leading to reconnection and flaring.

The numbers required for accretion or for electrodynamic models are generally reasonable. Specifically, if $L$ is the energy yield to be provided, accretion requires

$$
\frac{\mathrm{M}}{\mathrm{M}_{\odot} \mathrm{yr}^{-1}}=\frac{\mathrm{L}}{10^{47} \operatorname{erg~s}^{-1}}
$$

In the case of electrodynamic models

$$
\frac{\mathrm{B}}{100 \mathrm{G}}=\left(\frac{\mathrm{L}}{10^{47} \mathrm{erg} \mathrm{s}^{-1}}\right)^{1 / 2} \frac{10^{16} \mathrm{~cm}}{\mathrm{R}}
$$

Here we shall not enter into the details of accretion. We stress, however, that the standard approach followed in connection with thermal $\mathrm{X}$-ray sources cannot be adopted without major modifications. As is well 
known, the usual thermal disks would radiate mostly in the UV range because of their low temperature

$$
\mathrm{T}=510^{7}\left(\frac{\mathrm{M}_{\odot}}{\mathrm{M}}\right)^{1 / 4}{ }^{\circ} \mathrm{K} .
$$

One is therefore led to consider alternative ways to heat the infalling plasma and/or the outflowing radiation, such as the possible occurrence of flares dissipating magnetic energy (e.g., Maraschi et al. 1979) and the comptonisation of the low-energy photons. Even so, in a thermal scenario it appears rather difficult to explain the emission of hard $\mathrm{X}$-rays and of gamma rays (up to $100 \mathrm{MeV}$ ) as observed from 3C 273 (Swanenburg et a1. 1978). The latter would require a gas at $10^{12}{ }^{\circ} \mathrm{K}$ over a region of size $10^{17}-10^{18} \mathrm{~cm}$, very far from the central body (Cavallo and Rees 1978). It is much more likely that in most cases there is a combination of thermal and nonthermal phenomena. Something similar occurs also in the galactic source Sco $X-1$ where the $X$-ray emission extends into the hard region and where there is definite evidence for an associated nonthermal radio emission.

In the following, we shall consider the basic features of the electrodynamic models and in particular those aspects which are geometry-independent.

\section{THE ELECTRODYNAMIC MODELS}

Various authors have considered AGN models leading to electrodynamic outflows. Some of them involve the large scale equivalent of pulsars (Cavaliere et al. 1969; Morrison 1969; Woltjer 1971); others deal with electro-optical cascades near black holes (Blandford and Znajek 1977; Thorne and Blandford, this conference); still others can be applied both to spinars and black holes (see, e.g., Lovelace 1976). The common feature is that a large region of space is envisaged where the particles can be accelerated in situ by electromagnetic fields anchored to a central supermassive object. In this way one overcomes two major well-known difficulties with the theory of extreme nonthermal envi ronments :

(a) The very short lifetime of the electrons against radiation losses. In this case the lifetime has only a formal meaning because the particles are re-accelerated continuously by the same fields in which they radiate.

(b) The low density of thermal gas compatible with the polarization measurements, e.g., in BL Lac sources (Blandford and Rees 1978). This demands a continuous stirring of the same particles, rather than the steady injection of new high-energy electrons which would then decay into thermal ones. 
In a previous paper (Pacini and Salvati 1978), we have shown that the physical conditions in the active region can be reasonably constrained by means of general considerations, without resorting to detailed modeling. Indeed, the power radiated across the e.m. spectrum should match the energy carried by the electrodynamic outflow (see eq. 2 ) if the efficiency of the acceleration process is close to 1 . The further conversion from particle energy to photon energy is always very efficient due to the short radiated lifetime. Also, one naturally expects emission in (at least) two different frequency ranges, one associated with the synchrtron process

$$
\nu_{s}=10^{6} \quad B \quad \gamma^{2} \mathrm{~Hz}
$$

and the other corresponding to the simultaneous self-Compton process

$$
v_{i c}=\gamma^{2} v_{s}
$$

The typical parameters derived from the above equations--if one assumes $L \simeq 10^{47}$ erg $\mathrm{s}^{-1}$ and $R \simeq 10^{16} \mathrm{~cm}-$ are $\mathrm{B} \simeq 10^{2} \mathrm{G}, \gamma \simeq 10^{2}-10^{3}$, in good agreement with recent experimental results (Bregman et al. $1981 \mathrm{a}, \mathrm{b})$.

It is also worthwhile to compare the theoretical expectations with the observational evidence concerning the broadband distribution of the emitted radiation. The optical emission should be accompanied by inverse Compton X-rays of comparable intensity: This is because--as a consequence of eq. 2--the synchrotron photon energy density is of the same order as the energy density of the primary e.m. field. The prediction is consistent with the general observational trend (Tananbaum et a1. 1979; Setti and Woltjer 1979). The inverse Compton emission would be less important than the synchrotron emission only in case of strongly anisotropic sources. In our opinion, the measured ratio $\mathrm{L}_{\mathrm{X}} / \mathrm{L}_{\mathrm{opt}} \simeq 1$ represents one of the most important clues as to the merits of electrodynamic models. Higher order inverse Compton emission could have a comparable intensity, but it is more likely that the output is severely affected by the reduced Klein-Nishina crosssection and by internal reabsorption processes (Cavallo and Rees (1979).

In addition to the global condition that the energy flow should be conserved, one can also consider the possibility that in the active region the energy attained by the individual particles is limited by the radiative reaction. If so, one should write

$$
\text { e } v E=\frac{B^{2}}{4 \pi} \quad c \sigma_{T} \gamma^{2}
$$

where $E=B$ and $v$ is the particle drive velocity along $E$ (the instantaneous velocity is of course $=c$ ). 
Equation 6 entails a characteristic frequency $\nu_{\max }=\left(\mathrm{e}^{2} / \mathrm{mc}^{2}\right)\left(\mathrm{c} / \sigma_{\mathrm{T}}\right)$ $\simeq 50 \mathrm{MeV}$, provided that $\mathrm{v}$ is set equal to $\mathrm{c}$ on purely dimensional grounds. It is worthwhile to note that this frequency represents a natural upper limit to the spectrum emitted via synchrotron radiation in any loss-limited model. It is, however, unlikely that most particles will reach the limiting energy, and this should lead to the observed much softer spectrum.

\section{CONCLUSIONS}

Although progress in our understanding of the prime mover in active galactic nuclei has been slower than one may have hoped, we have now reasonable evidence that the energy source is associated with a supermassive configuration which has undergone gravitational collapse down to the black hole stage or its proximity. The choice between accretion and electrodynamic models cannot yet be made, but models of the latter kind seem able to account for the general energy budget and the gross spectral distribution. The parameters required for the strength of the e.m. field (such as, for instance, $B \simeq 10^{2}-10^{3} \mathrm{G}$ over distances $10^{15}-10^{16} \mathrm{~cm}$ ) are the same as those inferred from recent observations (Bregman et al. 1981 a,b). Many details of the acceleration process, radiation transport, formation of jets and connection with the large-scale activity must still be worked out and at present their analysis is at a preliminary stage.

This work has been supported partly by the National Research Council (CNR) and partly by NATO Grant 1946. One of us (F.P.) is indebted to the Institute of Astronomy, Cambridge, UK for hospitality during the writing of this paper.

\section{REFERENCES}

Ange1, J.R.P., and Stockman, H.S. 1980, Ann. Rev. Astron. Astrophys. 18,321 .

Blandford, R. D., and Rees, M. J. 1978, Pittsburgh Conference on BL Lac Objects, ed. A. M. Wolfe, p. 328 .

Blandford, R. D., and Znajek, R. L. 1977, M.N.R.A.S. 179, 433.

Bregman, J. N., et al. 1981a, preprint.

Bregman, J. N., et a1. 1981b, preprint.

Burbidge, G. R. $\overline{1956,}$ Astrophys. J. 124, 416.

Cavaliere, A., Pacini, F., and Setti, G. 1969, Astrophys. Lett. 4, 103. Cava11o, G., and Rees, M. J. 1978, M.N.R.A.S. 183, 359 .

Ginzburg, V. L., and Ozernoy, L. M. 1977, Astrophys. Space Sci. 50, 23. Jones, T. W., and Burbidge, G. R. 1973, Astrophys. J. 186, 791. Lovelace, R.V.E. 1976, Nature 262, 649.

Maraschi, L., Perola, G. C., Reina, C., and Treves, A. 1979, Astrophys. J. 230, 243 .

Morrison, P. 1969, Astrophys. J. 157, L73.

Pacini, F., and Salvati, M. 1978, Astrophys. J. 225, L99. 
Rees, M. J. 1980, Proceedings of the 1980 Texas Symposium on Relativistic Astrophysics, Baltimore, in press.

Salpeter, E. E. 1964, Astrophys. J. 140, 796.

Setti, G., and Woltjer, L. 1979, Ast $\overline{r o n}$. Astrophys. 76, L1.

Stein, W. A., O'De11, S. L., and Strittmatter, P. A. 1976, Ann. Rev. Astron. Astrophys. 14, 173 .

Swanenburg, B. N., et al. 1978, Nature 275, 298.

Tananbaum, H., et a $\cdot 1979$, Astrophys. J. 234, L9.

Woltjer, L. 1971 , Nuclei of Galaxies, ed. O'Conne11, Pontificiae Academiae Scripta Varia 35, 477.

\section{DISCUSSION}

REES: To be stable against bifiburcation, fragmentation, etc., a spinar must be pressure-supported along the rotation axis. This pressure will come predominantly from radiation. Therefore, any spinar must emit thermal radiation at the Eddington limit. This rules out spinars as the power source in most double radio sources (when a large mass is required on energy grounds, but high nuclear thermal luminosity is not seen). Spinars are perhaps--if they ever form--no more than short-lived processors of massive black holes. 